\title{
Guidance Law for Near Space Interceptor based on Block Backstepping Sliding Mode and Extended State Observer
}

\section{GUO Chao*}

School of Automation, Northwestern Polytechnical University, Xi'an, China

\section{LIANG Xiao-Geng**}

Luoyang Optoelectro Technology Development Center, Luoyang, China

\begin{abstract}
This paper proposes a novel guidance law based on the block backstepping sliding mode control and extended state observer (ESO), which also takes into account the autopilot dynamic characteristics of the near space interceptor (NSI), and the impact angle constraint of attacking the maneuvering target . Based on the backstepping control approach, the target maneuvers and the parameter uncertainties of the autopilot are regarded as disturbances of the outer loop and inner loop, respectively. Then, the ESO is constructed to estimate the target acceleration and the inner loop disturbance, and the block backstepping sliding model guidance law is employed, based on the estimated disturbance value. Furthermore, in order to avoid the "explosion of complexity" problem, first-order low-pass filters are also introduced, to obtain differentiations of the virtual control variables. The stability of the closed-loop guidance system is also proven, based on the Lyapunov theory. Finally, simulation results demonstrate that the proposed guidance law can not only overcome the influence of the autopilot dynamic delay and target maneuvers, but also obtain a small miss distance.
\end{abstract}

Key words: Guidance law, Extended state observer (ESO), Backstepping sliding mode control, Near space interceptor (NSI)

\section{Introduction}

The near space interceptor (NSI) with aerodynamic fins and reaction jets is an effective defense scheme to deal with the threat of near space air-breathing hypersonic vehicles (NSHV) [1], and the terminal guidance law is the key technology of the near space interception system. The proportional navigation guidance (PNG) law is the most popularly used guidance law for the task of intercepting a weakly maneuvering target, or a non-maneuvering target [2]. However, PNG may result in an unacceptable miss distance for intercepting a target with larger maneuvering capability. The augmented PNG (APNG) can obtain high guidance precision, by introducing target acceleration compensation, which is often difficult to obtain and estimate.

The autopilot lag of the NSI usually has significant influence on the guidance precision, especially in the presence of a larger maneuvering target $[4,5]$. Therefore, it is necessary to take into account the autopilot lag in the guidance law design; and an effective method to do this is to use a low-order model (first-order or second-order) to approximate the autopilot dynamics. On the other hand, in order to maximize the lethality of the warheads, the proposed guidance law should not only ensure the interceptor has a small miss distance, but also hit the target at an appropriate attitude $[6,7]$. The guidance system with the autopilot dynamics and the impact angle constraints satisfies the block lower-triangular structure , which includes the matched and unmatched disturbances [8]. Therefore, backstepping control is a natural choice to deal with unmatched disturbances, but the problem of "explosion of complexity" caused by repeated differentiations of the virtual control variables is inevitable. This problem can be
This is an Open Access article distributed under the terms of the Creative Commons Attribution Non-Commercial License (http://creativecommons.org/licenses/bync/3.0/) which permits unrestricted non-commercial use, distribution, and reproduction in any medium, provided the original work is properly cited. (cc) * Ph. D Student, Corresponding author : dongda20052742@163.com ** Professor 
solved by introducing first-order low-pass filters [9].

To intercept a larger maneuvering target, some advanced control methods have also been applied to the guidance law, such as the nonlinear $H_{\infty}$ guidance law $[10,11]$, and sliding mode guidance (SMG) law [12, 13]. However, the above methods dealt with the influence of target maneuvers at the price of sacrificing normal guidance performance. In Ref. [10], the guidance law design is formulated as a nonlinear $H_{\infty}$ disturbance attenuation problem, in which the target accelerations are regarded as unpredictable disturbances. It is also well known that $H_{\infty}$ control $[10,11]$ is a passive feedback regulation method that efficiently attenuates the effect of system disturbances, and only rejects disturbances under a given attenuation level, rather than completely removing them from the guidance system. Moreover, the $H_{\infty}$ guidance law relies on the solution of the HamiltonJacobi-Isaacs (HJI) partial differential equation, which is difficult to solve, except for simple or special cases. SMG is an efficient method to deal with the influence of the target maneuver and parameter uncertainties, but chattering is an unavoidable problem of engineering application [12]. The saturation function and boundary layer method are often used to alleviate chattering, at the price of disturbance rejection performance.

Extended state observer-based control (ESOBC) provides an active disturbance rejection method, and has been successfully applied in many fields, such as the MAGLEV system [14], permanent magnet synchronous motor (PMSM) [15], and so on [16]. In the framework of the ESOBC, a baseline controller is firstly designed, under the assumption that there are no system disturbances, such as dynamic inversion control, and sliding mode control; then, the extended state observer (ESO) is developed to estimate the system disturbances [17], and the feed-forward compensation term based on the estimated value is introduced, to remove the influence of the disturbances.

Motivated by the aforementioned considerations, this paper dealt with the guidance law design problem for the NSI based on block backstepping sliding mode control and ESO. The target maneuvers and the parameter uncertainties of the autopilot are regarded as disturbances of the outer loop and inner loop, respectively. Then, a novel block backstepping sliding mode guidance law is developed, based on the estimated disturbance value. Finally, simulation results show the effectiveness of the proposed guidance scheme.

Briefly, the rest of this paper is organized as follows. Section 2 introduces the guidance model description for the NSI. Section 3 presents the guidance law, based on the block backstepping sliding mode and ESO. Section 4 gives the simulation results for the NSI guidance system. Finally, a brief conclusion is drawn in Section 5 .

\section{Guidance model description of the NSI}

Assuming that the interceptor and target are point masses, the relative motion geometry between the NSI and the target is shown in Fig. 1, where $M$ and $T$ represent the mass center of the interceptor and target, respectively. Oxyz and $O x_{4} y_{4} z_{4}$ denote the inertial and line-of-sight (LOS) coordinate system, respectively. $q_{\varepsilon}$ and $q_{\beta}$ are the azimuth angle and elevation angle in the LOS coordinate system, respectively. $r$ is the relative distance between the interceptor and target.

As shown in Fig. 1, the dynamics of the missile-target motion in three-dimensional space can be described as follows:

$$
\begin{aligned}
& \ddot{r}-r \dot{q}_{\varepsilon}^{2}-r \dot{q}_{\beta}^{2} \cos ^{2} q_{\varepsilon}=a_{t r}-a_{m r} \\
& r \ddot{q}_{\varepsilon}+2 \dot{r} \dot{q}_{\varepsilon}+r \dot{q}_{\beta}^{2} \sin q_{\varepsilon} \cos q_{\varepsilon}=a_{t \varepsilon}-a_{m \varepsilon} \\
& -r \ddot{q}_{\beta} \cos q_{\varepsilon}-2 \dot{r} \dot{q}_{\beta} \cos q_{\varepsilon}+2 r \dot{q}_{\varepsilon} \dot{q}_{\beta} \sin q_{\varepsilon}=a_{t \beta}-a_{m \beta}
\end{aligned}
$$

where, $\left[a_{m r}, a_{m \varepsilon}, a_{m \beta}\right]^{T}$ and $\left[a_{t r}, a_{t \varepsilon}, a_{t \beta}\right]^{T}$ are the interceptor and target accelerations in the LOS coordinate system, respectively.

In addition, the autopilot dynamics of the NSI can be approximately described by the following second-order model:

$$
\begin{aligned}
& \ddot{a}_{m \varepsilon}=-2 \zeta \omega_{n} \dot{a}_{m \varepsilon}-\omega_{n}^{2} a_{m \varepsilon}+\omega_{n}^{2} a_{m \varepsilon}^{c}+d_{\varepsilon 4} \\
& \ddot{a}_{m \beta}=-2 \zeta \omega_{n} \dot{a}_{m \beta}-\omega_{n}^{2} a_{m \beta}+\omega_{n}^{2} a_{m \beta}^{c}+d_{\beta 4}
\end{aligned}
$$

where, $a_{m \varepsilon}^{c}$ and $a_{m \beta}^{c}$ denote the acceleration command of the NSI in the vertical and lateral plane, respectively; $\zeta$ and $\omega_{n}$ denote the damping ratio and natural frequency of the NSI autopilot, respectively; and $d_{\varepsilon 4}$ and $d_{\beta 4}$ are the disturbances and parameter uncertainties in the autopilot loop, respectively.

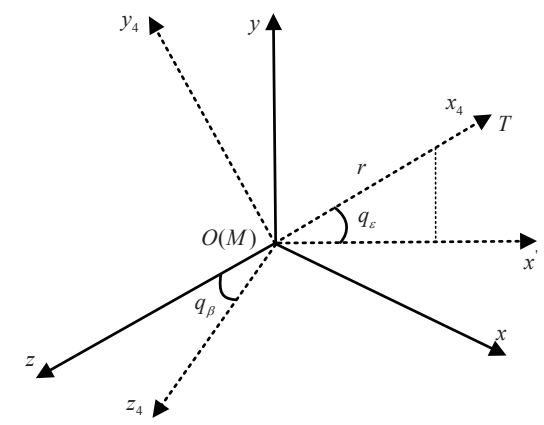

Fig. 1. Three-dimensional interception geometry 
The attack angle denotes the angle between the interceptor velocity vector and the target velocity vector in the guidance terminal time. Based on [6], the attack angle constraint can be transformed into the constraint problem of the terminal LOS angle:

$$
q_{\varepsilon}\left(t_{f}\right)=q_{\varepsilon d}, q_{\beta}\left(t_{f}\right)=q_{\beta d}
$$

where, $t_{f}$ is the guidance terminal time, and $q_{\varepsilon d}$ and $q_{\beta d}$ are the desired terminal LOS angle in the vertical and lateral plane, respectively.

In practical applications, the acceleration along the interceptor's velocity cannot be controlled in the terminal guidance processes. Therefore, we only need to discuss the relative motion normal to the LOS, and design the acceleration command to send the LOS angular rate to zero, or to a small neighborhood of zero.

Define the variables $\boldsymbol{x}_{1} \triangleq\left[q_{\varepsilon}-q_{\varepsilon d}, q_{\beta}-q_{\beta d}\right]^{T}$, $\boldsymbol{x}_{2} \triangleq\left[\dot{q}_{\varepsilon}, \dot{q}_{\beta}\right]^{T}, \boldsymbol{x}_{3} \triangleq\left[a_{m \varepsilon}, a_{m \beta}\right]^{T}, \boldsymbol{x}_{4} \triangleq\left[\dot{a}_{m \varepsilon}, \dot{a}_{m \beta}\right]^{T}$, $\boldsymbol{u} \triangleq\left[a_{m \varepsilon}^{c}, a_{m \beta}^{c}\right]^{T}, \boldsymbol{a}_{t} \triangleq\left[a_{t \varepsilon},-a_{t \beta}\right]^{T}$, and $\boldsymbol{d}_{4} \triangleq\left[d_{\varepsilon 4}, d_{\beta 4}\right]^{T}$. The guidance system (1) (6) can then be written in the following form:

$$
\left\{\begin{array}{l}
\dot{x}_{1}=x_{2} \\
\dot{x}_{2}=f_{2}\left(x_{1}, x_{2}\right)+g_{21}\left(x_{1}\right) x_{3}+g_{22}\left(x_{1}\right) a_{t} \\
\dot{x}_{3}=x_{4} \\
\dot{x}_{4}=f_{4}\left(x_{3}, x_{4}\right)+g_{4} u+d_{4} \\
y=\left[x_{1}, x_{2}\right]^{T}
\end{array}\right.
$$

where,

$$
\begin{aligned}
& \boldsymbol{f}_{2}\left(\boldsymbol{x}_{1}, \boldsymbol{x}_{2}\right) \triangleq\left[\begin{array}{c}
-\frac{2 \dot{r}}{r} \dot{q}_{\varepsilon}-\dot{q}_{\beta}^{2} \sin q_{\varepsilon} \cos q_{\varepsilon} \\
-\frac{2 \dot{r}}{r} \dot{q}_{\beta}+2 \dot{q}_{\varepsilon} \dot{q}_{\beta} \tan q_{\varepsilon}
\end{array}\right], \boldsymbol{g}_{21}\left(\boldsymbol{x}_{1}\right) \triangleq\left[\begin{array}{cc}
\frac{-1}{r} & 0 \\
0 & \frac{1}{r \cos q_{\varepsilon}}
\end{array}\right], \boldsymbol{g}_{22}\left(\boldsymbol{x}_{1}\right) \triangleq\left[\begin{array}{cc}
\frac{1}{r} & 0 \\
0 & \frac{1}{r \cos q_{\varepsilon}}
\end{array}\right], \\
& \boldsymbol{f}_{4}\left(\boldsymbol{x}_{3}, \boldsymbol{x}_{4}\right) \triangleq\left[\begin{array}{l}
-2 \zeta \omega_{n} \dot{a}_{m s}-\omega_{n}^{2} a_{m s} \\
-2 \zeta \omega_{n} \dot{a}_{m \beta}-\omega_{n}^{2} a_{m \beta}
\end{array}\right], \boldsymbol{g}_{4} \triangleq\left[\begin{array}{cc}
\omega_{n}^{2} & 0 \\
0 & \omega_{n}^{2}
\end{array}\right] .
\end{aligned}
$$

Assumption 1. It is assumed that the elements of $\boldsymbol{g}_{21}\left(\boldsymbol{x}_{1}\right)$ and $\boldsymbol{g}_{21}^{-1}\left(\boldsymbol{x}_{1}\right)$ are all bounded, which will be used in the stability analysis of the closed-loop guidance system.

Remark 1. The guidance and control system will stop working, when the relative distance satisfies $r<r_{f}$, where $r_{f}$ is a positive constant. Therefore, assumption 1 is reasonable.

Therefore, the design objective in this paper is to design a guidance law such that the guidance law can not only obtain a small miss distance, but also guarantee the NSI has an appropriate attitude to intercept the maneuvering target, i.e., design an appropriate controller for the time-varying system (7), to make the absolute value of the output as small as possible, in spite of the disturbances.

\section{Guidance law design and stability analysis}

In this section, a novel guidance law is developed for the time-varying system (7), based on the block backstepping sliding mode control and ESO; and the stability analysis of the closed-loop system is also given.

\subsection{Guidance law design}

The compensation term based on sliding mode control is often used to deal with the target maneuver influence on the guidance system. However, strong robustness of the SMG is obtained by sacrificing the normal guidance performance, and the high frequency chattering also limits the engineering application of the SMG. Therefore, the target maneuver is regarded as the outer loop disturbance of the guidance system in this paper, and the estimated disturbance value derived by the ESO is employed as the feed-forward compensation term, to remove the influence of the target maneuver on the guidance system.

Define the variable $\tilde{\boldsymbol{x}}_{2}=\left[v_{\varepsilon}, v_{\beta}\right]^{T} \triangleq\left[r \dot{q}_{\varepsilon}, r \dot{q}_{\beta} \cos q_{\varepsilon}\right]^{T}$. We can obtain the following system:

$$
\dot{\tilde{\boldsymbol{x}}}_{2}=\tilde{\boldsymbol{f}}_{2}\left(\boldsymbol{x}_{1}, \tilde{\boldsymbol{x}}_{2}\right)+\tilde{\boldsymbol{g}}_{21} \boldsymbol{x}_{3}+\boldsymbol{a}_{t}
$$

where,

$$
\tilde{\boldsymbol{f}}_{2}\left(\boldsymbol{x}_{1}, \tilde{\boldsymbol{x}}_{2}\right) \triangleq\left[\begin{array}{c}
-\frac{v_{r} v_{\varepsilon}}{r}-\frac{v_{\beta}^{2} \tan q_{\varepsilon}}{r} \\
-\frac{v_{r} v_{\beta}}{r}+\frac{v_{\varepsilon} v_{\beta} \tan q_{\varepsilon}}{r}
\end{array}\right], \quad \tilde{\boldsymbol{g}}_{21} \triangleq\left[\begin{array}{cc}
-1 & 0 \\
0 & 1
\end{array}\right] .
$$

Then, the following second-order ESO is proposed to estimate the target acceleration $a_{t}$ :

$$
\left\{\begin{array}{c}
\overline{\boldsymbol{e}}_{21}=\overline{\boldsymbol{z}}_{21}-\tilde{\boldsymbol{x}}_{2}, \overline{\boldsymbol{e}}_{22}=\overline{\boldsymbol{z}}_{22}-\boldsymbol{a}_{t}, \overline{\boldsymbol{z}}_{22}=\hat{\boldsymbol{a}}_{t} \\
\dot{\overline{\boldsymbol{z}}}_{21}=\overline{\boldsymbol{z}}_{22}-\mu_{21} \overline{\boldsymbol{e}}_{21}+\tilde{\boldsymbol{f}}_{2}\left(\boldsymbol{x}_{1}, \tilde{\boldsymbol{x}}_{2}\right)+\tilde{\boldsymbol{g}}_{21} \boldsymbol{x}_{3} \\
\dot{\overline{\boldsymbol{z}}}_{22}=-\mu_{22}\left[\operatorname{fal}\left(\bar{e}_{211}, \alpha_{21}, \delta_{21}\right),\right. \\
\left.\operatorname{fal}\left(\bar{e}_{212}, \alpha_{22}, \delta_{22}\right)\right]^{T}
\end{array}\right.
$$

where, $\overline{\boldsymbol{e}}_{21}$ and $\overline{\boldsymbol{e}}_{22}$ are the estimation errors of the ESO, $\overline{\boldsymbol{z}}_{21}$ and $\overline{\boldsymbol{z}}_{22}$ are the observer outputs, and $\mu_{21}$ and $\mu_{22}$ are the observer gain coefficients. The function $f a l(\bullet)$ is defined as the following form:

$$
f a l\left(\bar{e}_{21 i}, \alpha_{2 i}, \delta_{2 i}\right)=\left\{\begin{array}{ll}
\left|\bar{e}_{21 i}\right|^{\alpha_{2 i}} \operatorname{sgn}\left(\bar{e}_{21 i}\right) & \left|\bar{e}_{21 i}\right|>\delta_{2 i} \\
\bar{e}_{21 i} / \delta_{2 i}^{1-\alpha_{2 i}} & \left|\bar{e}_{21 i}\right| \leq \delta_{2 i}
\end{array}, \quad i \in \aleph \triangleq\{1,2\},\right.
$$

with $0<\alpha_{2 i}<1$ and $\delta_{2 i}>0, i \in \aleph$. For the appropriate parameter values of $\mu_{21}, \mu_{22}, \alpha_{2 i}$ and $\delta_{2 i}, i \in \mathfrak{\aleph}$, the observer output $\overline{\boldsymbol{z}}_{21}$ converges to $\tilde{\boldsymbol{x}}_{2}$, and $\overline{\boldsymbol{z}}_{22}$ converges to $\boldsymbol{a}_{t}$. The stability analysis of the ESO (9) can be seen in [17]. 
The observer error dynamics can be written as:

$$
\left\{\begin{array}{c}
\dot{\overline{\boldsymbol{e}}}_{21}=\overline{\boldsymbol{e}}_{22}-\mu_{21} \overline{\boldsymbol{e}}_{21} \\
\dot{\overline{\boldsymbol{e}}}_{22}=-\dot{\boldsymbol{a}}_{t}-\mu_{22}\left[\operatorname{fal}\left(\bar{e}_{211}, \alpha_{21}, \delta_{21}\right),\right. \\
\left.\operatorname{fal}\left(\bar{e}_{212}, \alpha_{22}, \delta_{22}\right)\right]^{T}
\end{array}\right.
$$

where, $\overline{\boldsymbol{e}}_{21} \triangleq\left[\bar{e}_{211}, \bar{e}_{212}\right]^{T}, \overline{\boldsymbol{e}}_{22} \triangleq\left[\bar{e}_{221}, \bar{e}_{222}\right]^{T}$ and $\dot{\boldsymbol{a}}_{t} \triangleq\left[\dot{a}_{t 1}, \dot{a}_{t 2}\right]^{T}$.

From (10), we can obtain:

$$
\begin{aligned}
& \left\{\begin{array}{l}
\left|\bar{e}_{21 i}\right|=\left|\dot{a}_{t i} / \mu_{22}\right|^{1 / \alpha_{2 i}} \\
\left|\bar{e}_{22 i}\right|=\mu_{21}\left|\dot{a}_{t i} / \mu_{22}\right|^{1 / \alpha_{2 i}}
\end{array} \text {, if }\left|\bar{e}_{21 i}\right|>\delta_{2 i}\right. \\
& \left\{\begin{array}{l}
\left|\bar{e}_{21 i}\right|=\left|\dot{a}_{t i} \delta_{2 i}^{1-\alpha_{2 i}}\right| / \mu_{22} \\
\left|\bar{e}_{22 i}\right|=\mu_{21}\left|\dot{a}_{t i} \delta_{2 i}^{1-\alpha_{2 i}}\right| / \mu_{22}
\end{array}, \text { if }\left|\bar{e}_{21 i}\right| \leq \delta_{2 i}\right.
\end{aligned}
$$

From (11) and (12), we can know that the estimation errors of the ESO can be forced small enough, by selecting the appropriate parameters $\mu_{21}, \mu_{22}, \alpha_{2 i}$ and $\delta_{2 i}, i \in \mathfrak{\aleph}$. The proposed ESO can effectively estimate the system state $\tilde{\boldsymbol{x}}_{2}$ and the target acceleration $a_{t}$, respectively. Furthermore, the parameter $\mu_{22}$ can be selected large enough to make $\left|\dot{a}_{t i} / \mu_{22}\right|$ as small as possible, and $\mu_{21}$ and $\alpha_{2 i}$ should also be small enough, such that the estimation errors of the target acceleration are small enough.

Based on the same method, the following ESO is proposed, to estimate the disturbances in the autopilot loop:

$$
\left\{\begin{array}{c}
\overline{\boldsymbol{e}}_{41}=\overline{\boldsymbol{z}}_{41}-\boldsymbol{x}_{4}, \overline{\boldsymbol{e}}_{42}=\overline{\boldsymbol{z}}_{42}-\boldsymbol{d}_{4}, \overline{\boldsymbol{z}}_{42}=\hat{\boldsymbol{d}}_{4} \\
\dot{\overline{\boldsymbol{z}}}_{41}=\overline{\boldsymbol{z}}_{42}-\mu_{41} \overline{\boldsymbol{e}}_{41}+\boldsymbol{f}_{4}\left(\boldsymbol{x}_{3}, \boldsymbol{x}_{4}\right)+\boldsymbol{g}_{4} \boldsymbol{u} \\
\dot{\overline{\boldsymbol{z}}}_{42}=-\mu_{42}\left[\operatorname{fal}\left(\bar{e}_{411}, \alpha_{41}, \delta_{41}\right),\right. \\
\text { fal } \left.\left(\bar{e}_{412}, \alpha_{42}, \delta_{42}\right)\right]^{T}
\end{array}\right.
$$

where, $\overline{\boldsymbol{e}}_{41}$ and $\overline{\boldsymbol{e}}_{42}$ are the estimation errors of the ESO. The function $\mathrm{fal}(\bullet)$ is defined as follows:

$$
f a l\left(\bar{e}_{41 i}, \alpha_{4 i}, \delta_{4 i}\right)=\left\{\begin{array}{ll}
\left|\bar{e}_{41 i}\right|^{\alpha_{4 i}} \operatorname{sgn}\left(\bar{e}_{41 i}\right) & \left|\bar{e}_{41 i}\right|>\delta_{4 i} \\
\bar{e}_{41 i} / \delta_{4 i}^{1-\alpha_{4 i}} & \left|\bar{e}_{41 i}\right| \leq \delta_{4 i}
\end{array}, \quad i \in \mathfrak{\aleph} .\right.
$$

Based on the system (7), and the above ESO (9) and (13), we consider the following guidance law:

Step 1: Define the first error surface as follows:

$$
\boldsymbol{s}_{2}=\boldsymbol{x}_{2}+\frac{V_{m}}{r} \boldsymbol{k}_{1} \boldsymbol{x}_{1}
$$

where, $V_{m}$ is the velocity of the NSI, and $k_{1}=\operatorname{diag}\left\{k_{11}, k_{12}\right\}$ is a positive-definite matrix. The derivative of $s_{2}$ is:

$$
\dot{\boldsymbol{s}}_{2}=\boldsymbol{f}_{2}\left(\boldsymbol{x}_{1}, \boldsymbol{x}_{2}\right)+\boldsymbol{g}_{21}\left(\boldsymbol{x}_{1}\right) \boldsymbol{x}_{3}+\boldsymbol{g}_{22}\left(\boldsymbol{x}_{1}\right) \boldsymbol{a}_{t}+\frac{V_{m}}{r} \boldsymbol{k}_{1} \boldsymbol{x}_{2}-\frac{V_{m} \dot{r}}{r^{2}} \boldsymbol{k}_{1} \boldsymbol{x}_{1}
$$

Then, the following virtual control $\boldsymbol{x}_{3}^{*}$ is selected, to make $s_{2}$ converge to zero:

$$
\boldsymbol{x}_{3}^{*}=-\boldsymbol{g}_{21}^{-1}\left(\boldsymbol{x}_{1}\right)\left[\boldsymbol{f}_{2}\left(\boldsymbol{x}_{1}, \boldsymbol{x}_{2}\right)+\boldsymbol{g}_{22}\left(\boldsymbol{x}_{1}\right) \hat{\boldsymbol{a}}_{t}+\frac{V_{m}}{r} \boldsymbol{k}_{1} \boldsymbol{x}_{2}-\frac{V_{m} \dot{r}}{r^{2}} \boldsymbol{k}_{1} \boldsymbol{x}_{1}+\boldsymbol{k}_{2} \boldsymbol{s}_{2}\right]
$$

where $k_{2}=\operatorname{diag}\left\{k_{21}, k_{22}\right\}$ is the gain matrix. The following firstorder low-pass filter is proposed to obtain the filtered virtual control $\overline{\boldsymbol{x}}_{3}^{*}$ :

$$
\tau_{3} \dot{\bar{x}}_{3}^{*}+\overline{\boldsymbol{x}}_{3}^{*}=\boldsymbol{x}_{3}^{*}, \overline{\boldsymbol{x}}_{3}^{*}(0)=\boldsymbol{x}_{3}^{*}(0)
$$

where, $\tau_{3}=\operatorname{diag}\left\{\tau_{31}, \tau_{32}\right\}$ denotes the filter time constant matrix.

Step 2: Denote the second error surface as follows:

$$
s_{3}=x_{3}-\bar{x}_{3}^{*}
$$

and its derivative is:

$$
\dot{\boldsymbol{s}}_{3}=\boldsymbol{x}_{4}-\dot{\overline{\boldsymbol{x}}}_{3}^{*}
$$

Then, the following virtual control $\boldsymbol{x}_{4}^{*}$ is selected, to make $\boldsymbol{s}_{3}$ converge to zero:

$$
\boldsymbol{x}_{4}^{*}=-\boldsymbol{k}_{3} \boldsymbol{s}_{3}+\dot{\overline{\boldsymbol{x}}}_{3}^{*}
$$

where, $k_{3}=\operatorname{diag}\left\{k_{31}, k_{32}\right\}$ is the gain matrix. The following filter is proposed to obtain the filtered virtual control $\overline{\boldsymbol{x}}_{4}^{*}$ :

$$
\tau_{4} \dot{\bar{x}}_{4}^{*}+\overline{\boldsymbol{x}}_{4}^{*}=\boldsymbol{x}_{4}^{*}, \overline{\boldsymbol{x}}_{4}^{*}(0)=\boldsymbol{x}_{4}^{*}(0)
$$

where, $\tau_{4}=\operatorname{diag}\left\{\tau_{41}, \tau_{42}\right\}$ denotes the filter time constant matrix.

Step 3: Denote the third error surface as follows:

$$
s_{4}=x_{4}-\bar{x}_{4}^{*}
$$

and its derivative is:

$$
\dot{\boldsymbol{s}}_{4}=\boldsymbol{f}_{4}\left(\boldsymbol{x}_{3}, \boldsymbol{x}_{4}\right)+\boldsymbol{g}_{4} \boldsymbol{u}+\boldsymbol{d}_{4}-\dot{\overline{\boldsymbol{x}}}_{4}^{*}
$$

Then, we can design the following actual control to make $s_{4}$ converge to zero:

$$
\boldsymbol{u}=\boldsymbol{g}_{4}^{-1}\left[-\boldsymbol{f}_{4}\left(\boldsymbol{x}_{3}, \boldsymbol{x}_{4}\right)-\hat{\boldsymbol{d}}_{4}+\dot{\overline{\boldsymbol{x}}}_{4}^{*}-\boldsymbol{k}_{4} \boldsymbol{s}_{4}-\boldsymbol{k}_{5} \operatorname{sig}\left(\boldsymbol{s}_{4}\right)^{\lambda_{4}}\right]
$$

where, $\operatorname{sig}\left(s_{4}\right)^{\lambda_{4}}=\left[\operatorname{sgn}\left(s_{41}\right)\left|s_{41}\right|^{\lambda_{41}}, \operatorname{sgn}\left(s_{42}\right)\left|s_{42}\right|^{\lambda_{42}}\right]^{T}, \lambda_{4}=\operatorname{diag}\left\{\lambda_{41}, \lambda_{42}\right\}$, $\boldsymbol{k}_{4}=\operatorname{diag}\left\{k_{41}, k_{42}\right\}$ and $\boldsymbol{k}_{5}=\operatorname{diag}\left\{k_{51}, k_{52}\right\}$ are the positivedefinite matrices.

Remark 2. If the filter time constant matrices satisfy the constraints $\tau_{3}=\tau_{4}=0$, we have $\overline{\boldsymbol{x}}_{3}^{*}=\boldsymbol{x}_{3}^{*}$ and $\overline{\boldsymbol{x}}_{4}^{*}=\boldsymbol{x}_{4}^{*}$, and should compute the differentiation of the virtual control in the backstepping control design process. In this paper, we introduce two first-order low-pass filters, to compute the derivatives of the virtual control, i.e., $\dot{\bar{x}}_{3}^{*}=-\tau_{3}^{-1}\left(\bar{x}_{3}^{*}-x_{3}^{*}\right)$ and $\dot{\overline{\boldsymbol{x}}}_{4}^{*}=-\tau_{4}^{-1}\left(\overline{\boldsymbol{x}}_{4}^{*}-\boldsymbol{x}_{4}^{*}\right)$. 
Remark 3. The NSI acceleration is achieved by transferring $u$ from the LOS coordinates system into the inertial coordinate system.

\subsection{Stability analysis}

Assumption 2. It is assumed that the estimated errors of $a_{t}$ and $d_{4}$ satisfy the following constraints:

$$
\left\|\overline{\boldsymbol{e}}_{22}\right\| \leq N_{2}, \quad\left\|\overline{\boldsymbol{e}}_{42}\right\| \leq N_{4}
$$

where, $N_{2}$ and $N_{4}$ are positive constants.

Based on the above analysis, we have the following theorem:

Theorem 1. Consider the uncertain nonlinear system (7), and let Assumption 1 hold. If the design parameters $\boldsymbol{k}_{i}(i \in\{2,3,4,5\})$ are selected large enough, and $\tau_{i}(i \in\{3,4\})$ are selected small enough, the proposed guidance law based on the block backstepping sliding mode control and ESO can keep all the states of the closed-loop system bounded, and the LOS angle rate arbitrarily small.

The proof of Theorem 1 can be seen in Appendix A.

Remark 4. In order to obtain a small miss distance, we usually choose the parameters $\boldsymbol{k}_{i}(i \in\{2,3,4,5\})$ large enough, and $\tau_{i}(i \in\{3,4\})$ small enough. However, this may lead to unacceptable transient responses and large acceleration command. Also, the filter time constants $\tau_{i}$ cannot be made arbitrarily small in practice. Therefore, in the design process we should balance the tradeoff between the transient responses and the guidance accuracy.

Remark 5. During the implementation, the acceleration of the NSI is bounded, according to the following saturation function:

$$
u_{i}= \begin{cases}u_{i \max } \operatorname{sgn}\left(u_{i}\right) & \text { if }\left|u_{i}\right| \geq u_{i \max } \\ u_{i} & \text { if }\left|u_{i}\right|<u_{i \max }\end{cases}
$$

where, $u_{i \max }, i \in \aleph$ is the maximum acceleration of the interceptor.

A structural diagram of the guidance system can be seen in Fig. 2.

\section{Simulation results}

In this section, some numerical simulations for the NSI guidance system will be given, to demonstrate the effectiveness of the proposed guidance scheme. The initial conditions of the NSI and target in the inertial coordinate system are shown in Table 1.

Table 1. Initial conditions of the NSI and target

\begin{tabular}{cccc}
\hline Parameter & Value & Parameter & Value \\
\hline$x_{m 0}$ & $0 \mathrm{~m}$ & $x_{t 0}$ & $15204.6 \mathrm{~m}$ \\
$y_{m 0}$ & $0 \mathrm{~m}$ & $y_{t 0}$ & $6840.4 \mathrm{~m}$ \\
$z_{m 0}$ & $0 \mathrm{~m}$ & $z_{t 0}$ & $11046.8 \mathrm{~m}$ \\
$V_{m 0}$ & $1500 \mathrm{~m} / \mathrm{s}$ & $V_{t 0}$ & $1700 \mathrm{~m} / \mathrm{s}$ \\
$\theta_{m 0}$ & $30^{\circ}$ & $\theta_{t 0}$ & $-10^{\circ}$ \\
$\psi_{v m 0}$ & $-30^{\circ}$ & $\psi_{v t 0}$ & $140^{\circ}$ \\
\hline
\end{tabular}

The NSI with aerodynamic fins and reaction jets is used to deal with the threat of the NSHV, so the response time of the acceleration command is about $0.15 \sim 0.2 \mathrm{~s}$. Therefore, the damping ratio and natural frequency of the NSI autopilot are selected as $\zeta=0.8$ and $\omega_{n}=20$, respectively.

The parameters of the ESO (9) and (13) are given as follows:

$$
\begin{aligned}
& \mu_{21}=50, \mu_{22}=150, \alpha_{21}=\alpha_{22}=0.2, \delta_{21}=\delta_{22}=0.001, \\
& \mu_{41}=5, \mu_{42}=200, \alpha_{41}=\alpha_{42}=0.2, \delta_{41}=\delta_{42}=0.01 .
\end{aligned}
$$

The parameters of the proposed guidance law are selected as follows:

$$
\begin{aligned}
& k_{1}=\operatorname{diag}\{2,2\}, k_{1}=k_{3}=\operatorname{diag}\{10,10\}, k_{4}=\operatorname{diag}\{40,40\}, \\
& k_{5}=\operatorname{diag}\{20,20\}, \\
& \lambda=\operatorname{diag}\{0.6,0.6\}, \tau_{3}=\operatorname{diag}\{0.01,0.01\}, \\
& \tau_{4}=\operatorname{diag}\{0.025,0.025\} .
\end{aligned}
$$

The maximized acceleration of the NHSV (X-43 and $\mathrm{X}-51 \mathrm{~A}$ ) is about $2 \sim 4 \mathrm{~g}$. The interception conditions in this paper are then selected, so that the acceleration of the target

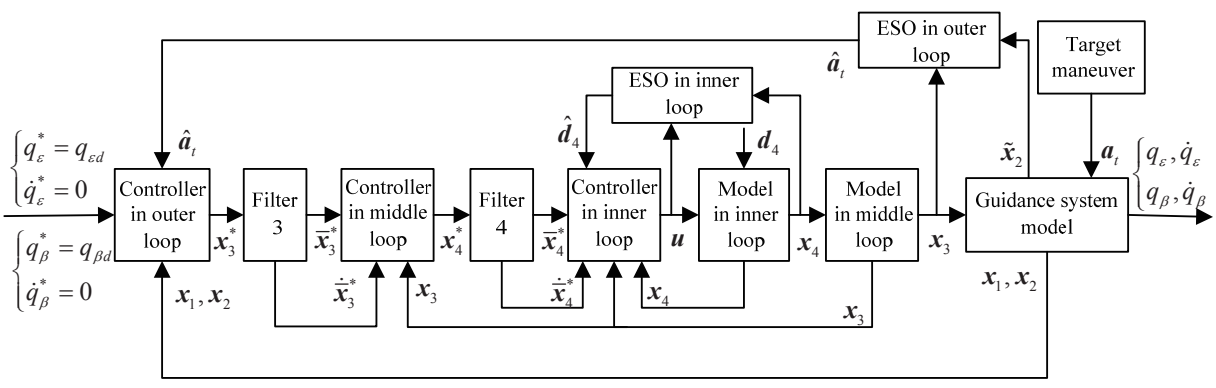

Fig. 2. Structural diagram of the guidance system 
is $a_{t \varepsilon}=a_{t \beta}=40 \sin (t) \mathrm{m} / \mathrm{s}^{2}$, and the disturbances of the autopilot loop are $d_{\varepsilon 4}=200 \sin (t)$ and $d_{\beta 4}=200 \cos (t)$. The flight path angle and heading angle of the target at the guidance terminal time are $\theta_{t}\left(t_{f}\right)=190^{\circ}$ and $\psi_{v t}\left(t_{f}\right)=140^{\circ}$ , and the desired attack angles in the vertical and lateral plane are $-156.5^{\circ}$ and $-173^{\circ}$; then by some calculation, we can obtain the desired LOS angles as $q_{\varepsilon}=21.1^{\circ}$ and $q_{\beta}=-37.1^{\circ}$.

The proposed guidance law based on the block backstepping sliding mode control and ESO (denoted by BBSMG+ESO, Case 4) is firstly applied to the NSI guidance system, and the PNG (Case 1), SMG (Case 2), and APNG (Case 3) are also studied for the simulation comparisons.

The PNG is given as follows:

$$
u_{\varepsilon}=-N \dot{r} \dot{q}_{\varepsilon}, u_{\beta}=N \dot{r} \dot{q}_{\beta}
$$

where, the proportional coefficient is $N=3.2$.

The SMG is given as follows:

$$
u_{\varepsilon}=-\bar{N} \dot{r} \dot{q}_{\varepsilon}+\varepsilon \operatorname{sgn}\left(\dot{q}_{\varepsilon}\right), u_{\beta}=\bar{N} \dot{r} \dot{q}_{\beta}-\varepsilon \operatorname{sgn}\left(\dot{q}_{\beta}\right)
$$

where, $\bar{N}=4.5$ and $\varepsilon=200$.

The APNG is also given, as follows:

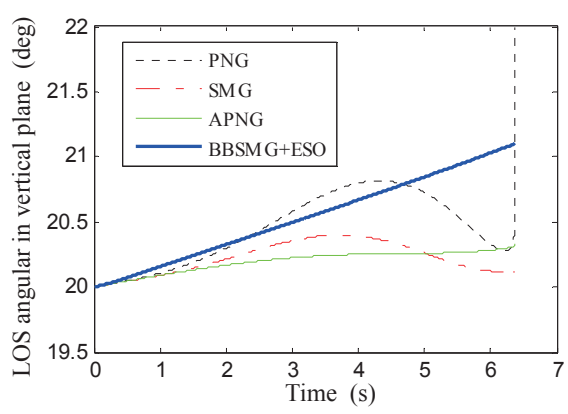

(a) LOS angular in the vertical plane

Fig. 3. Curves of the LOS angular

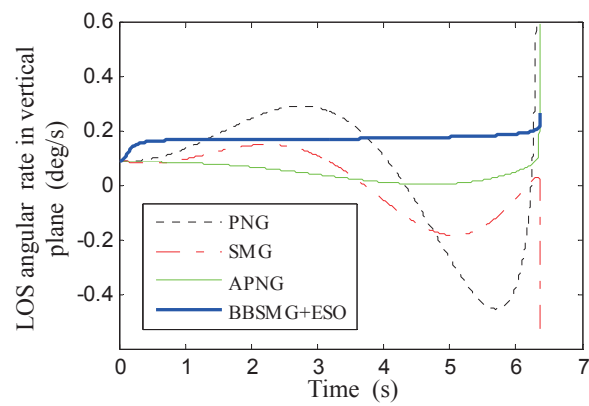

(a) LOS angular rate in the vertical plane

$$
u_{\varepsilon}=-N \dot{r} \dot{q}_{\varepsilon}+a_{t \varepsilon}, \quad u_{\beta}=N \dot{r} \dot{q}_{\beta}+a_{t \beta}
$$

The response curves of the LOS angle and angle rate based on the four guidance schemes are shown in Fig. 3 and Fig. 4. Under the same simulation condition, the proposed guidance scheme for Case 4 can make the LOS angle and angle rate converge to the steady state value more quickly, and the transient value of the system response is also small. The actual and estimated value response curves of the target acceleration and the autopilot loop disturbance are shown in Fig. 5 and Fig. 6 , and we observe that the proposed ESO can efficiently estimate the target acceleration and the autopilot loop disturbance. Fig. 7 shows the response curves of the NSI acceleration command, based on the four guidance schemes. Fig. 8 shows the movement paths of the NSI and the target in the inertial coordinate system.

To illustrate the robustness to target maneuver and external disturbance, a Monte Carlo simulation study consisting of 100 sample runs is carried out. The simulation result comparisons of the four guidance schemes are shown in Table 2, in terms of miss distance and interception time. We can see from Table 2 that the miss distance for Case 4 is minimal.

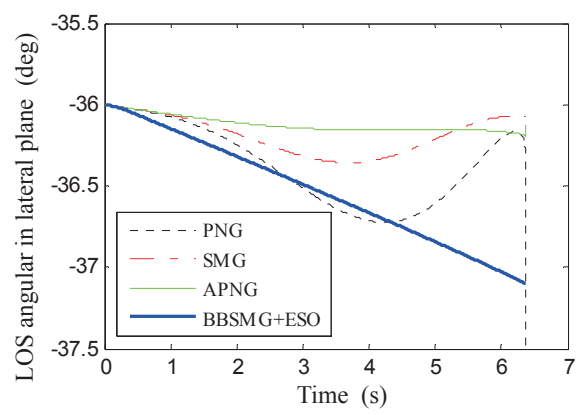

(b) LOS angular in the lateral plane

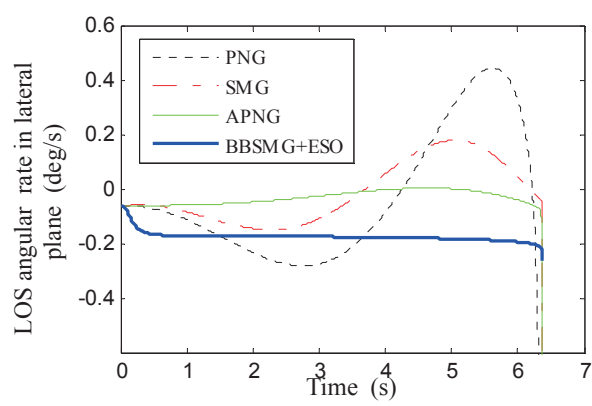

(b) LOS angular rate in the lateral plane

Fig. 4. Curves of the LOS angular rate 


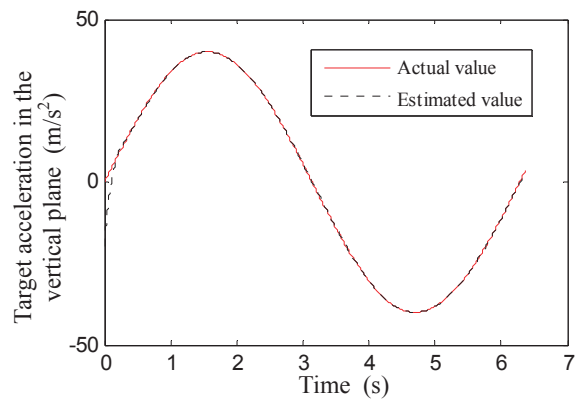

(a) Target acceleration in the vertical plane

Fig. 5. Curves of the target acceleration

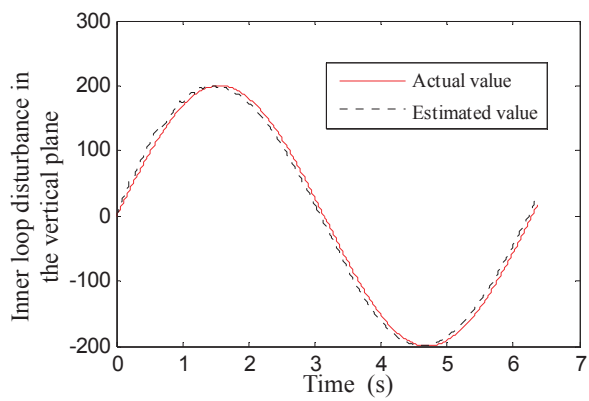

(a) Inner loop disturbance in the vertical plane

Fig. 6. Curves of the inner loop disturbance

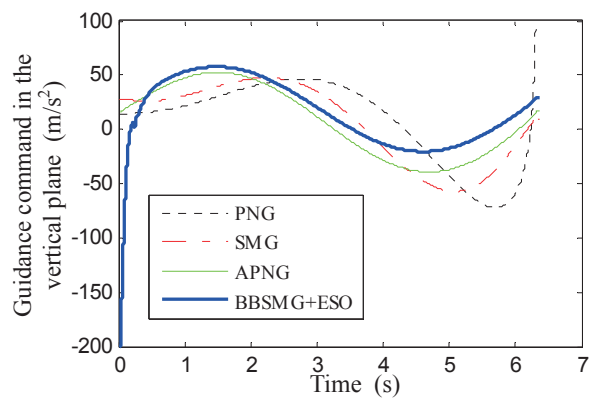

(a) Guidance command in the vertical plane

Fig. 7. Guidance command based on the four guidance schemes

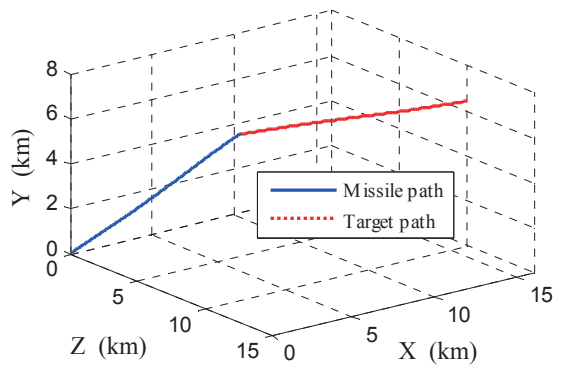

Fig. 8. Trajectories of the interceptor and target

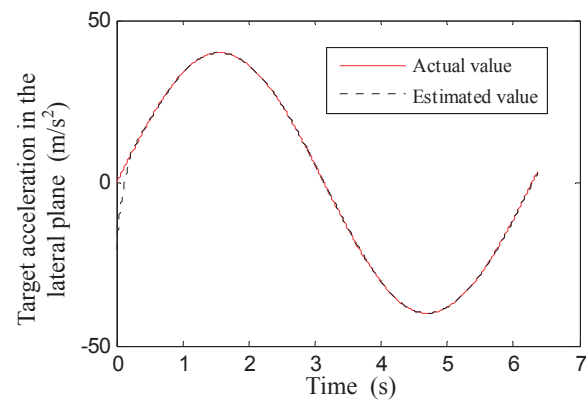

(b) Target acceleration in the lateral plane

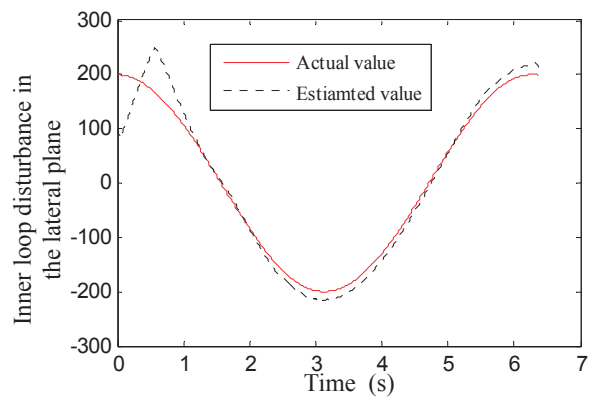

(b) Inner loop disturbance in the lateral plane

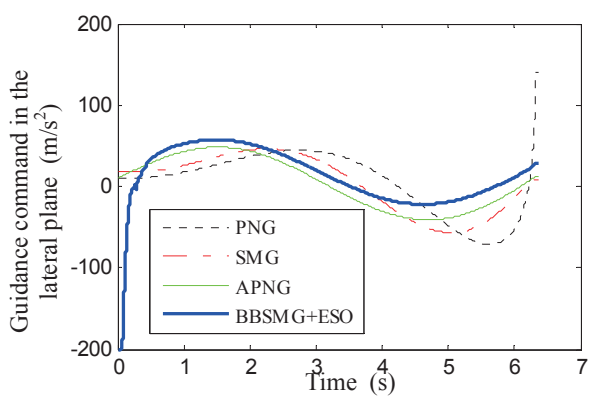

(b) Guidance command in the lateral plane

Table 2. Guidance performance comparison of the four schemes

Guidance method $\quad$ Miss Distance $(\mathrm{m}) \quad$ Interception Time $(\mathrm{s})$

$\begin{array}{ccc}\text { PNG } & 0.8439 \mathrm{~m} & 6.369 \\ \text { SMG } & 0.6961 \mathrm{~m} & 6.368 \\ \text { APNG } & 0.3965 \mathrm{~m} & 6.368 \\ \text { BSMG+ESO } & 0.0348 \mathrm{~m} & 6.368\end{array}$




\section{Conclusion}

In this paper, a novel guidance law is proposed for the NSI, based on block backstepping sliding mode control and ESO. Firstly, the guidance system with the autopilot dynamic can be divided into the outer loop, middle loop and inner loop. Then, the ESO is developed to estimate the target acceleration of the outer loop and the disturbance of the inner loop, and the estimated values are employed as feed-forward compensations, to remove the influence of total system disturbances. Furthermore, first-order low-pass filters are also introduced, to compute the differentiations of the virtual control variables at each step of the block backstepping sliding mode guidance law. Finally, simulation results demonstrate that the proposed guidance law can obtain a small miss distance, compared with the other guidance schemes.

\section{Appendix A: Proof of Theorem 1}

Define the following filter errors:

$$
\boldsymbol{y}_{3}=\overline{\boldsymbol{x}}_{3}^{*}-\boldsymbol{x}_{3}^{*}, \boldsymbol{y}_{4}=\overline{\boldsymbol{x}}_{4}^{*}-\boldsymbol{x}_{4}^{*}
$$

then, the dynamics of the filter error can be written as follows:

$$
\dot{y}_{3}=-\tau_{3}^{-1} y_{3}-\dot{x}_{3}^{*}, \quad \dot{y}_{4}=-\tau_{4}^{-1} y_{4}-\dot{x}_{4}^{*}
$$

From (14), (18), (22) and (A1), we have:

$$
\begin{aligned}
& \boldsymbol{x}_{2}=\boldsymbol{s}_{2}-\frac{V_{m}}{r} \boldsymbol{k}_{1} \boldsymbol{x}_{1} \\
& \boldsymbol{x}_{3}=\boldsymbol{s}_{3}+\overline{\boldsymbol{x}}_{3}^{*}=\boldsymbol{s}_{3}+\boldsymbol{y}_{3}+\boldsymbol{x}_{3}^{*} \\
& \boldsymbol{x}_{4}=\boldsymbol{s}_{4}+\overline{\boldsymbol{x}}_{4}^{*}=\boldsymbol{s}_{4}+\boldsymbol{y}_{4}+\boldsymbol{x}_{4}^{*}
\end{aligned}
$$

From (7) and (14) (24), we can obtain that:

$$
\begin{aligned}
\dot{\boldsymbol{s}}_{2} & =\dot{x}_{2}+\frac{V_{m}}{r} \boldsymbol{k}_{1} \dot{\boldsymbol{x}}_{1}-\frac{V_{m} \dot{r}}{r^{2}} \boldsymbol{k}_{1} \boldsymbol{x}_{1} \\
& =\dot{\boldsymbol{x}}_{2}+\frac{V_{m}}{r} \boldsymbol{k}_{1} \boldsymbol{x}_{2}-\frac{V_{m} \dot{r}}{r^{2}} \boldsymbol{k}_{1} \boldsymbol{x}_{1} \\
& =\boldsymbol{f}_{2}\left(\boldsymbol{x}_{1}, \boldsymbol{x}_{2}\right)+\boldsymbol{g}_{21}\left(\boldsymbol{x}_{1}\right)\left(\boldsymbol{s}_{3}+\boldsymbol{y}_{3}+\boldsymbol{x}_{3}^{*}\right)+\boldsymbol{g}_{22}\left(\boldsymbol{x}_{1}\right) \boldsymbol{a}_{t}+\frac{V_{m}}{r} \boldsymbol{k}_{1} \boldsymbol{x}_{2}-\frac{V_{m} \dot{r}}{r^{2}} \boldsymbol{k}_{1} \boldsymbol{x}_{1} \\
& =\boldsymbol{g}_{21}\left(\boldsymbol{x}_{1}\right)\left(\boldsymbol{s}_{3}+\boldsymbol{y}_{3}\right)-\boldsymbol{k}_{2} \boldsymbol{s}_{2}+\boldsymbol{g}_{22}\left(\boldsymbol{x}_{1}\right) \boldsymbol{a}_{t}-\boldsymbol{g}_{22}\left(\boldsymbol{x}_{1}\right) \hat{\boldsymbol{a}}_{t} \\
& =\boldsymbol{g}_{21}\left(\boldsymbol{x}_{1}\right)\left(\boldsymbol{s}_{3}+\boldsymbol{y}_{3}\right)-\boldsymbol{k}_{2} \boldsymbol{s}_{2}-\boldsymbol{g}_{22}\left(\boldsymbol{x}_{1}\right) \overline{\boldsymbol{e}}_{22} \\
\dot{\boldsymbol{s}}_{3} & =\dot{\boldsymbol{x}}_{3}-\dot{\overline{\boldsymbol{x}}}_{3}^{*}=\boldsymbol{x}_{4}-\dot{\overline{\boldsymbol{x}}}_{3}^{*} \\
& =\boldsymbol{s}_{4}+\boldsymbol{y}_{4}+\boldsymbol{x}_{4}^{*}-\dot{\overline{\boldsymbol{x}}}_{3}^{*} \\
& =\boldsymbol{s}_{4}+\boldsymbol{y}_{4}-\boldsymbol{k}_{3} \boldsymbol{s}_{3}
\end{aligned}
$$

and,

$$
\begin{aligned}
\dot{\boldsymbol{s}}_{4} & =\dot{\boldsymbol{x}}_{4}-\dot{\overline{\boldsymbol{x}}}_{4}^{*} \\
& =\boldsymbol{f}_{4}\left(\boldsymbol{x}_{3}, \boldsymbol{x}_{4}\right)+\boldsymbol{g}_{4} \boldsymbol{u}+\boldsymbol{d}_{4}-\dot{\overline{\boldsymbol{x}}}_{4}^{*} \\
& =-\boldsymbol{k}_{4} \boldsymbol{s}_{4}-\boldsymbol{k}_{5} \operatorname{sig}\left(\boldsymbol{s}_{4}\right)^{\lambda_{4}}-\hat{\boldsymbol{d}}_{4}+\boldsymbol{d}_{4} \\
& =-\boldsymbol{k}_{4} \boldsymbol{s}_{4}-\boldsymbol{k}_{5} \operatorname{sig}\left(\boldsymbol{s}_{4}\right)^{\lambda_{4}}-\overline{\boldsymbol{e}}_{42}
\end{aligned}
$$

For the system (7), consider the following Lyapunov function:

$$
V=0.5\left(s_{2}^{T} s_{2}+s_{3}^{T} s_{3}+s_{4}^{T} s_{4}+y_{3}^{T} y_{3}+y_{4}^{T} y_{4}\right)
$$

The coefficients and variables for the system (7) and their derivatives are all bounded. Then by some simple calculations, we have:

$$
\begin{aligned}
& \left\|\dot{\boldsymbol{x}}_{3}^{*}\right\| \leq \eta_{3}\left(\boldsymbol{s}_{2}, \boldsymbol{s}_{3}, \boldsymbol{y}_{3}, \overline{\boldsymbol{e}}_{22}, \boldsymbol{k}_{2}\right) \\
& \left\|\dot{\boldsymbol{x}}_{4}^{*}\right\| \leq \eta_{4}\left(\boldsymbol{s}_{2}, \boldsymbol{s}_{3}, \boldsymbol{s}_{4}, \boldsymbol{y}_{3}, \boldsymbol{y}_{4}, \overline{\boldsymbol{e}}_{22}, \boldsymbol{k}_{2}, \boldsymbol{k}_{3}\right)
\end{aligned}
$$

where, $\eta_{3}$ and $\eta_{4}$ are both nonnegative continuous functions. For some given positive constant $R^{*}$, the following set:

$$
B_{r}=\left\{\left[\boldsymbol{s}_{2}^{T}, \boldsymbol{s}_{3}^{T}, \boldsymbol{s}_{4}^{T}, \boldsymbol{y}_{3}^{T}, \boldsymbol{y}_{4}^{T}, \overline{\boldsymbol{e}}_{22}^{T}\right]^{T}, V \leq R^{*}\right\}
$$

is compact. Therefore, $\eta_{3}$ and $\eta_{4}$ have maximum values on $B_{r}$, which satisfy the following constraints:

$$
\left\|\dot{\boldsymbol{x}}_{3}^{*}\right\| \leq M_{3},\left\|\dot{\boldsymbol{x}}_{4}^{*}\right\| \leq M_{4}
$$

where, $M_{3}$ and $M_{4}$ are some positive constants.

Then, by some simple computations, we have:

$$
\begin{aligned}
& \boldsymbol{s}_{2}^{T} \dot{\boldsymbol{s}}_{2} \boldsymbol{s}_{2}^{T} \boldsymbol{g}_{21}\left(\boldsymbol{s}_{3}+\boldsymbol{y}_{3}\right)-\boldsymbol{s}_{2}^{T} \boldsymbol{k}_{2} \boldsymbol{s}_{2}-\boldsymbol{s}_{2}^{T} \boldsymbol{g}_{22} \overline{\boldsymbol{e}}_{22} \\
& \leq 0.5 \boldsymbol{s}_{2}^{T} \boldsymbol{g}_{21}^{2} \boldsymbol{s}_{2}+0.5 \boldsymbol{s}_{3}^{T} \boldsymbol{s}_{3}+0.5 \boldsymbol{s}_{2}^{T} \boldsymbol{g}_{21}^{2} \boldsymbol{s}_{2}+0.5 \boldsymbol{y}_{3}^{T} \boldsymbol{y}_{3}-\boldsymbol{s}_{2}^{T} \boldsymbol{k}_{2} \boldsymbol{s}_{2}+0.5 \boldsymbol{s}_{2}^{T} \boldsymbol{g}_{22}^{2} \boldsymbol{s}_{2}+0.5 \boldsymbol{e}_{22}^{T} \overline{\boldsymbol{e}}_{22} \\
& \leq \boldsymbol{s}_{2}^{T}\left(\boldsymbol{g}_{21}^{2}-\boldsymbol{k}_{2}+0.5 \boldsymbol{g}_{22}^{2}\right) \boldsymbol{s}_{2}+0.5 \boldsymbol{s}_{3}^{T} \boldsymbol{s}_{3}+0.5 \boldsymbol{y}_{3}^{T} \boldsymbol{y}_{3}+0.5 N_{2}^{2} \\
& \boldsymbol{s}_{3}^{T} \dot{\boldsymbol{s}}_{3}=\boldsymbol{s}_{3}^{T}\left(\boldsymbol{s}_{4}+\boldsymbol{y}_{4}-\boldsymbol{k}_{3} \boldsymbol{s}_{3}\right) \\
& \quad \leq 0.5 \boldsymbol{s}_{3}^{T} \boldsymbol{s}_{3}+0.5 \boldsymbol{s}_{4}^{T} \boldsymbol{s}_{4}+0.5 \boldsymbol{s}_{3}^{T} \boldsymbol{s}_{3}+0.5 \boldsymbol{y}_{4}^{T} \boldsymbol{y}_{4}-\boldsymbol{s}_{3}^{T} \boldsymbol{k}_{3} \boldsymbol{s}_{3} \\
& \quad=\boldsymbol{s}_{3}^{T}\left(\boldsymbol{I}-\boldsymbol{k}_{3}\right) \boldsymbol{s}_{3}+0.5 \boldsymbol{s}_{4}^{T} \boldsymbol{s}_{4}+0.5 \boldsymbol{y}_{4}^{T} \boldsymbol{y}_{4} \\
& \boldsymbol{s}_{4}^{T} \dot{\boldsymbol{s}}_{4}=-\boldsymbol{s}_{4}^{T} \boldsymbol{k}_{4} \boldsymbol{s}_{4}-\boldsymbol{s}_{4}^{T} \boldsymbol{k}_{5} \operatorname{sig}\left(\boldsymbol{s}_{4}\right)^{\lambda_{4}}-\boldsymbol{s}_{4}^{T} \overline{\boldsymbol{e}}_{42} \\
& \leq-\boldsymbol{s}_{4}^{T} \boldsymbol{k}_{4} \boldsymbol{s}_{4}+k_{51}\left|s_{41}\right|^{\lambda_{41}+1}+k_{52}\left|s_{42}\right|_{42}^{\lambda_{42}+1}+0.5 \boldsymbol{s}_{4}^{T} \boldsymbol{s}_{4}+0.5 \overline{\boldsymbol{e}}_{42}^{T} \overline{\boldsymbol{e}}_{42} \\
& \leq-\boldsymbol{s}_{4}^{T} \boldsymbol{k}_{4} \boldsymbol{s}_{4}+k_{51}\left|s_{41}\right|\left(1+\mid s_{41}\right)+k_{52}\left|s_{42}\right|\left(1+\mid s_{42}\right)+0.5 \boldsymbol{s}_{4}^{T} \boldsymbol{s}_{4}+0.5 \overline{\boldsymbol{e}}_{42}^{T} \overline{\boldsymbol{e}}_{42} \\
& \leq-\boldsymbol{s}_{4}^{T} \boldsymbol{k}_{4} \boldsymbol{s}_{4}+k_{51}\left(0.5+1.5 s_{41}^{2}\right)+k_{52}\left(0.5+1.5 s_{42}^{2}\right)+0.5 \boldsymbol{s}_{4}^{T} \boldsymbol{s}_{4}+0.5 \boldsymbol{e}_{42}^{T} \overline{\boldsymbol{e}}_{42} \\
& \leq \boldsymbol{s}_{4}^{T}\left(0.5 \boldsymbol{I}-\boldsymbol{k}_{4}+1.5 \boldsymbol{k}_{5}\right) \boldsymbol{s}_{4}+0.5 N_{4}^{2}+0.5 k_{51}+0.5 k_{52}
\end{aligned}
$$




$$
\begin{aligned}
\boldsymbol{y}_{3}^{T} \dot{y}_{3} & =\boldsymbol{y}_{3}^{T}\left(-\tau_{3}^{-1} \boldsymbol{y}_{3}-\dot{\boldsymbol{x}}_{3}^{*}\right) \\
& \leq-\boldsymbol{y}_{3}^{T} \tau_{3}^{-1} \boldsymbol{y}_{3}+0.5 \boldsymbol{y}_{3}^{T} \boldsymbol{y}_{3}+0.5\left(\dot{\boldsymbol{x}}_{3}^{*}\right)^{T} \dot{\boldsymbol{x}}_{3}^{*} \\
& \leq \boldsymbol{y}_{3}^{T}\left(0.5 \boldsymbol{I}-\boldsymbol{\tau}_{3}^{-1}\right) \boldsymbol{y}_{3}+0.5 M_{3}^{2}
\end{aligned}
$$

and,

$$
\begin{aligned}
\boldsymbol{y}_{4}^{T} \dot{\boldsymbol{y}}_{4} & =\boldsymbol{y}_{4}^{T}\left(-\tau_{4}^{-1} \boldsymbol{y}_{4}-\dot{\boldsymbol{x}}_{4}^{*}\right) \\
& \leq-\boldsymbol{y}_{4}^{T} \tau_{4}^{-1} \boldsymbol{y}_{4}+0.5 \boldsymbol{y}_{4}^{T} \boldsymbol{y}_{4}+0.5\left(\dot{\boldsymbol{x}}_{4}^{*}\right)^{T} \dot{\boldsymbol{x}}_{4}^{*} \\
& \leq \boldsymbol{y}_{4}^{T}\left(0.5 \boldsymbol{I}-\tau_{4}^{-1}\right) \boldsymbol{y}_{4}+0.5 M_{4}^{2}
\end{aligned}
$$

By taking the time derivative of $V(t)$ along the solutions of the system (7), we have:

$$
\begin{aligned}
\dot{V}= & \boldsymbol{s}_{2}^{T} \dot{\boldsymbol{s}}_{2}+\boldsymbol{s}_{3}^{T} \dot{\boldsymbol{s}}_{3}+\boldsymbol{s}_{4}^{T} \dot{\boldsymbol{s}}_{4}+\boldsymbol{y}_{3}^{T} \dot{\boldsymbol{y}}_{3}+\boldsymbol{y}_{4}^{T} \dot{\boldsymbol{y}}_{4} \\
\leq & \boldsymbol{s}_{2}^{T}\left(\boldsymbol{g}_{21}^{2}-\boldsymbol{k}_{2}+0.5 \boldsymbol{g}_{22}^{2}\right) \boldsymbol{s}_{2}+0.5 \boldsymbol{s}_{3}^{T} \boldsymbol{s}_{3}+0.5 \boldsymbol{y}_{3}^{T} \boldsymbol{y}_{3}+0.5 N_{2}^{2} \\
& +\boldsymbol{s}_{3}^{T}\left(\boldsymbol{I}-\boldsymbol{k}_{3}\right) \boldsymbol{s}_{3}+0.5 \boldsymbol{s}_{4}^{T} \boldsymbol{s}_{4}+0.5 \boldsymbol{y}_{4}^{T} \boldsymbol{y}_{4} \\
& +\boldsymbol{s}_{4}^{T}\left(0.5 \boldsymbol{I}-\boldsymbol{k}_{4}+1.5 \boldsymbol{k}_{5}\right) \boldsymbol{s}_{4}+0.5 N_{4}^{2}+0.5 k_{51}+0.5 k_{52} \\
& +\boldsymbol{y}_{3}^{T}\left(0.5 \boldsymbol{I}-\boldsymbol{\tau}_{3}^{-1}\right) \boldsymbol{y}_{3}+0.5 M_{3}^{2}+\boldsymbol{y}_{4}^{T}\left(0.5 \boldsymbol{I}-\boldsymbol{\tau}_{4}^{-1}\right) \boldsymbol{y}_{4}+0.5 M_{4}^{2} \\
= & \boldsymbol{s}_{2}^{T}\left(\boldsymbol{g}_{21}^{2}-\boldsymbol{k}_{2}+0.5 \boldsymbol{g}_{22}^{2}\right) \boldsymbol{s}_{2}+\boldsymbol{s}_{3}^{T}\left(1.5 \boldsymbol{I}-\boldsymbol{k}_{3}\right) \boldsymbol{s}_{3}+\boldsymbol{s}_{4}^{T}\left(\boldsymbol{I}-\boldsymbol{k}_{4}+1.5 \boldsymbol{k}_{5}\right) \boldsymbol{s}_{4} \\
& +\boldsymbol{y}_{3}^{T}\left(\boldsymbol{I}-\boldsymbol{\tau}_{3}^{-1}\right) \boldsymbol{y}_{3}+\boldsymbol{y}_{4}^{T}\left(\boldsymbol{I}-\boldsymbol{\tau}_{4}^{-1}\right) \boldsymbol{y}_{4} \\
& +0.5 N_{2}^{2}+0.5 N_{4}^{2}+0.5 M_{3}^{2}+0.5 M_{4}^{2}+0.5 k_{51}+0.5 k_{52}
\end{aligned}
$$

If the designed parameters satisfy the following conditions:

$$
\left\{\begin{array}{l}
\boldsymbol{g}_{21}^{2}-\boldsymbol{k}_{2}+0.5 \boldsymbol{g}_{22}^{2} \leq-0.5 \kappa \boldsymbol{I} \\
1.5 \boldsymbol{I}-\boldsymbol{k}_{3} \leq-0.5 \kappa \boldsymbol{I} \\
\boldsymbol{I}-\boldsymbol{k}_{4}+1.5 \boldsymbol{k}_{5} \leq-0.5 \kappa \boldsymbol{I} \\
\boldsymbol{I}-\tau_{3}^{-1} \leq-0.5 \kappa \boldsymbol{I} \\
\boldsymbol{I}-\tau_{4}^{-1} \leq-0.5 \kappa \boldsymbol{I}
\end{array}\right.
$$

where $\kappa$ is a positive real number, then we have:

$$
\dot{V} \leq-\kappa V+C
$$

where, $C=0.5 N_{2}^{2}+0.5 N_{4}^{2}+0.5 M_{3}^{2}+0.5 M_{4}^{2}+0.5 k_{51}+0.5 k_{52}$.

By the comparison principle, it is easy to obtain that:

$$
V(t) \leq \frac{[\kappa V(0)-C] e^{-\kappa t}+C}{\kappa}
$$

The parameter $C / \kappa$ can be made arbitrarily small, by choosing $\kappa$ large enough. Therefore, $s_{2}, s_{3}, s_{4}, y_{3}$ and $y_{4}$ are all uniformly ultimately bounded, and we can both obtain the short miss distance, and guarantee the desired LOS angle.

\section{References}

[1] Dai, J., Cheng, J., and Guo, R., "Research on nearspace hypersonic weapon defense system and the key technology", Journal of the Academy of Equipment Command \& Technology, Vol. 21, No. 3, 2010, pp. 58-61.

[2] Zarchan, P., Tactical and strategic missile guidance, American Institute of Aeronautics and Astronautics, Reston, VA, 2012.

[3] Siouris, G., Missile guidance and control systems, Springer-Verlag, New York, 2004.

[4] Zhang, Z., Li, S., and Luo, S., "Composite guidance laws based on sliding mode control with impact angle constraint and autopilot lag", Transactions of the Institute of Measurement and Control, Vol. 35, No. 6, 2013, pp. 764-776.

[5] Qu, P., and Zhou, D., "A dimension reduction observerbased guidance law accounting for dynamics of missile autopilot", Proceedings of the Institution of Mechanical Engineers, Part G: Journal of Aerospace Engineering, Vol. 227, No. 7, 2013, pp. 1114-1121.

[6] Sun, S., Zhang, H., and Zhou, D., "Sliding mode guidance law with autopilot lag for terminal angle constrained trajectories", Journal of Astronautics, Vol. 34, No. 1, 2013, pp. 69-78.

[7] Zhang, Z., Li, S., and Luo, S., "Terminal guidance laws of missile based on ISMC and NDOB with impact angle constraint", Aerospace Science and Technology, Vol. 31, No. 1, 2013, pp. 30-41.

[8] Hou, M., Liang, X., and Duan, G., "Adaptive block dynamic surface control for integrated missile guidance and autopilot", Chinese Journal of Aeronautics, Vol. 26, No. 3, 2013, pp. 741-750.

[9] Swaroop, D., Hedrick, J., and Yip, P., et al., "Dynamic surface control for a class of nonlinear systems", IEEE Transactions on Automatic Control, Vol. 45, No. 10, 2000, pp. 1893-1899.

[10] Yang, C., and Chen, H., "Nonlinear $\mathrm{H} \infty$ guidance law for homing missiles", AIAA Journal of Guidance, Control, and Dynamics, Vol. 21, No. 6, 1998, pp. 882-890.

[11] Chen, B., Chen, Y., and Lin, C., "Nonlinear fuzzy $H_{\infty}$ guidance law with saturation of actuators against maneuvering targets", IEEE Transactions on Control Systems Technology, Vol. 10, No. 6, 2002, pp. 769-779.

[12] Zhou, D., Sun, S., and Teo, K., "Guidance laws with finite time convergence", AIAA Journal of Guidance, Control, and Dynamics, Vol. 32, No. 6, 2009, pp. 1838-1846.

[13] Zhou, D., New guidance laws for homing missile, National Defense Industry Press, Beijing, 2002.

[14] Li, S., Yang, J., and Chen, W., et al., "Generalized 
extended state observer based control for systems with mismatched uncertainties", IEEE Transactions on Industrial Electronics, Vol. 59, No. 12, 2012, pp. 4792- 4892.

[15] Liu, H., and Li, S., "Speed control for PMSM servo system using predictive functional control and extended state observer", IEEE Transactions on Industrial Electronics, Vol. 59, No. 2, 2012, pp. 1171- 1183.
[16] Xia, Y., and Fu, M., Compound control methodology for flight vehicles, Springer Science Business Media, Berlin, 2013.

[17] Han, J., Active disturbance rejection control technique--the technique for estimating and compensating the uncertainties, National Defense Industry Press, Beijing, 2008. 